\title{
Major duodenal papilla prolapse in Cronkhite-Canada syndrome
}

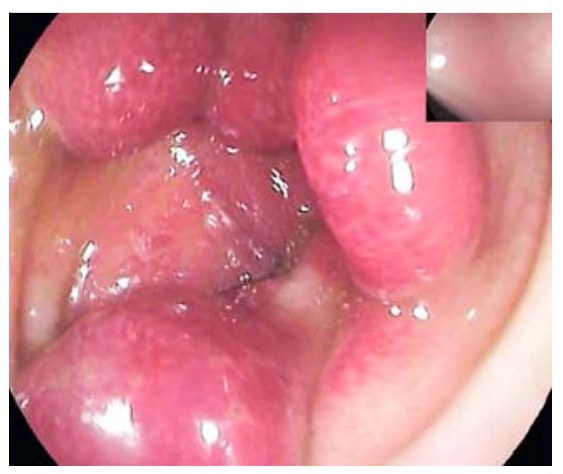

- Fig. 1 Cronkhite-Canada colonic polyposis in a 59-year-old Japanese woman with recurrent proctorrhagia and recently presenting alopecia, dysgeusia, and onychodystrophy.

We describe the case of a 59-year-old Japanese woman presenting with recurrent proctorrhagia. Her clinical history was unremarkable except for recently presenting alopecia, dysgeusia, and onychodystrophy.

Ileocolonoscopy showed multiple strawberry-like sessile polyps ranging from 5 to $20 \mathrm{~mm}$ in size in the rectosigmoid ( Fig. 1).

Upper endoscopy demonstrated hypertrophic gastric plicae and many sessile polyps of $5-15 \mathrm{~mm}$ in size spreading from the stomach to the distal duodenum ( Fig.2a,b). i-SCAN digital contrast (I-SCAN) and optical enhancement virtual chromoendoscopy (Optivista EPKi7010 video processor; Pentax, Tokyo, Japan) were activated to increase the detection of subtle mucosal changes, revealing several erosion-like mucosal defects within the surface of the polyps ( Video 1). During withdrawal, the dynamics of a major duodenal papillary prolapse were clearly observed within the context of a large, laterally spreading, superficial, and elevated polypoid projection ( $\triangleright$ Video 1 ).

Targeted biopsy samples showed histological features consistent with a diagnosis of Cronkhite-Canada syndrome ( Fig.3a,b). A capsule endoscopy ex-

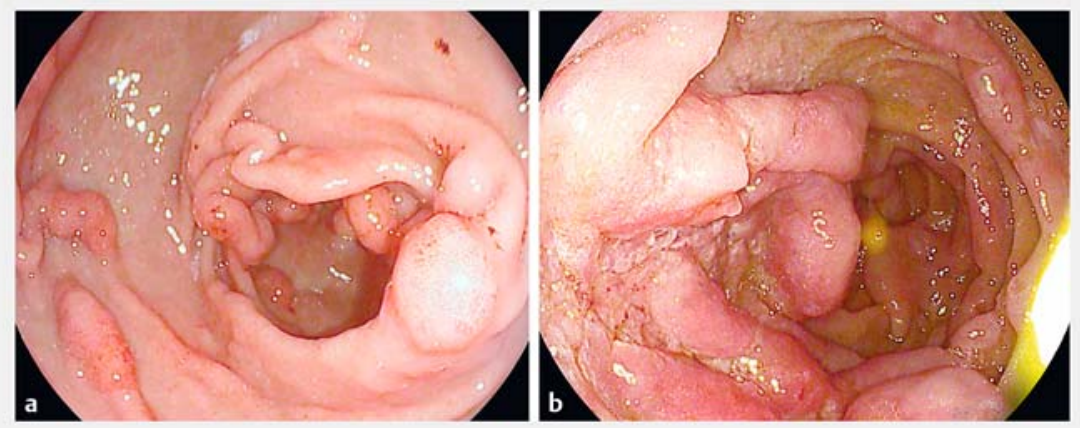

- Fig. 2 Cronkhite-Canada endoscopic appearances: a Hyperplastic plicae and polyposis of the stomach; $\mathbf{b}$ duodenal polyposis.

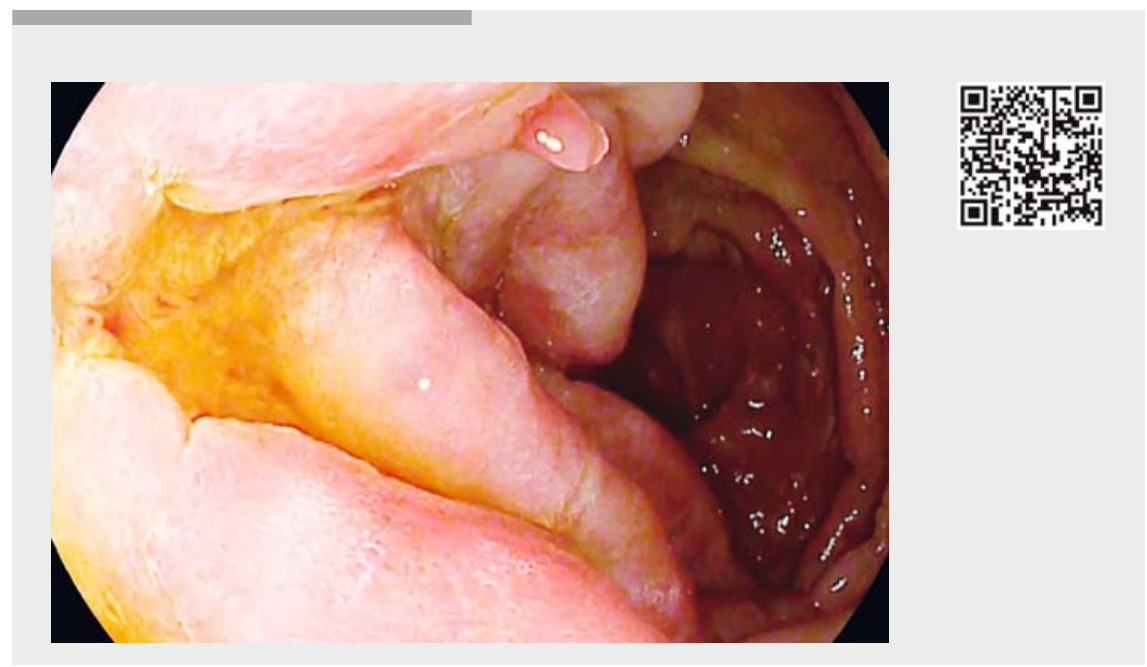

$\checkmark$ Video 1 Cronkhite-Canada syndrome. Enteroscopy conducted to the proximal jejunum, using high definition and optical enhancement virtual chromoendoscopy. A prolapsing major papilla is seen in duodenal polyposis.

cluded additional polyp locations and systemic steroid treatment was introduced to reduce both polyp formation and bleeding.

Cronkhite-Canada syndrome is an extremely rare nonhereditary gastrointestinal polyposis, with 450 cases reported in the literature, mainly in the Japanese population [1]. The syndrome usually presents with a triad of dermatologic disorders, including alopecia, onycodystrophy, and hyperpigmentation, associated with a variable degree of gastrointestinal symptoms including diarrhea, malabsorption, and hemorrhage [2]. Cronkhite-Canada polyps are usually sessile, with abundant stromal edema, hyperplastic glands, and cystic mucous retention, plus mild inflammation with predominant eosinophilic infiltrate within the surrounding mucosa [3]. The prognosis for Cronkhite-Canada syndrome is often unfavorable, because of complications (malabsorption, gastrointestinal 

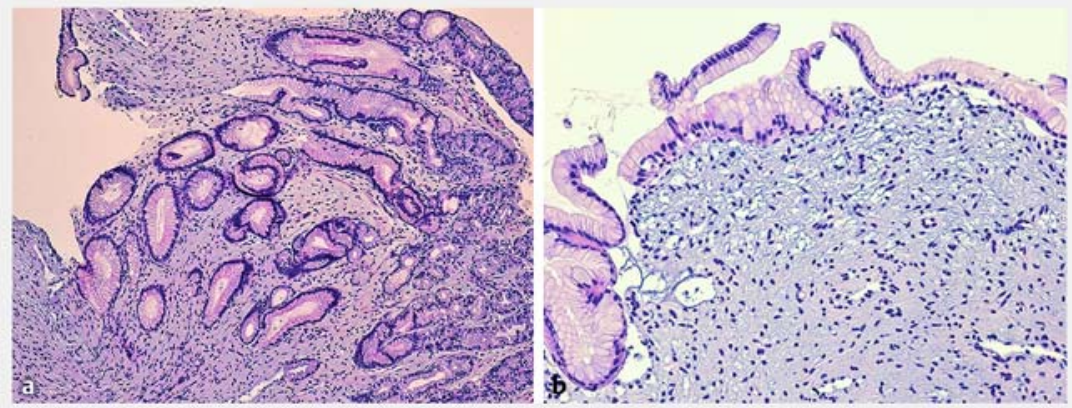

- Fig. 3 Histological appearances in Cronkhite-Canada syndrome: a gastric mucosa with hyperplastic glands without atypia (hematoxylin and eosin [H\&E], $\times 100$ ); b myxoid-edematous stroma with very scanty inflammatory infiltrates $(\mathrm{H} \& \mathrm{E}, \times 400)$.

hemorrhage, or intussusception) and the lack of standardized treatments [4]. The malignant transformation of polyps is still a matter of debate and no validated protocols for endoscopic surveillance are available [5].

Endoscopy_UCTN_Code_TTT_1AO_2AC

\section{Competing interests}

None

The authors

Beatrice Marinoni ${ }^{1}$, Gian E. Tontini ${ }^{1}$, Luca Elli ${ }^{1}$, Barbara Bruni ${ }^{2}$, Marco Maggioni ${ }^{3}$, Luca Pastorelli ${ }^{4,5}$, Maurizio Vecchi ${ }^{1,6}$

1 Gastroenterology and Endoscopy Unit, Fondazione IRCCS Ca' Granda Ospedale Maggiore Policlinico, Milan, Italy

2 Pathology and Cytodiagnostic Unit, IRCCS Policlinico San Donato, San Donato Milanese, Italy

3 Pathology Unit, Fondazione IRCCS Ca' Granda Ospedale Maggiore Policlinico, Milan, Italy
4 Gastroenterology and Digestive Endoscopy Unit, IRCCS Policlinico San Donato, San Donato Milanese, Italy

5 Department of Biomedical Sciences for Health, University of Milan, Milan, Italy

6 Department of Pathophysiology and Transplantation, University of Milan, Milan, Italy

\section{Corresponding author}

\section{B. Marinoni, MD}

Ospedale Maggiore Policlinico, Gastroenterology and Encoscopy Unit, Via commenda 12 milano, Milano Lombardia 20122, Italy

Fax: +39-2-55033414

bea_marinoni@hotmail.it

\section{References}

[1] Cronkhite LW Jr, Canada W]. Generalized gastrointestinal polyposis; an unusual syndrome of polyposis, pigmentation, alopecia and onychotrophia. N Engl J Med 1955; 252: 1011-1015

[2] Watanabe C, Komoto S, Tomita K et al. Endoscopic and clinical evaluation of treatment and prognosis of Cronkhite-Canada syndrome: a Japanese nationwide survey. J Gastroenterol 2016; 51: 327-336

[3] Zhao R, Huang M, Banafea O et al. CronkhiteCanada syndrome: a rare case report and literature review. BMC Gastroenterology 2016; 16: 23

[4] Yun SH, Cho JW, Kim JW et al. CronkhiteCanada syndrome associated with serrated adenoma and malignant polyp: a case report and a literature review of 13 CronkhiteCanada syndrome cases in Korea Clin Endosc 2013; 46: $301-305$

[5] Tontini GE, Rimondi A, Neumann $\mathrm{H}$ et al. Usefulness of virtual chromoendoscopy with optical enhancement in everyday clinical practice. Adv Res Gastroenterol Hepatol 2017. doi:10.19080/ARGH.2017.07.555719

\section{Bibliography}

DOI https://doi.org/10.1055/a-0800-8148

Published online: 7.2.2019

Endoscopy 2019; 51: E81-E82

(c) Georg Thieme Verlag KG

Stuttgart · New York

ISSN 0013-726X

\section{ENDOSCOPY E-VIDEOS}

https://eref.thieme.de/e-videos

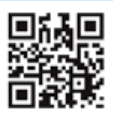

Endoscopy E-Videos is a free access online section, reporting on interesting cases and new

techniques in gastroenterological endoscopy. All papers include a high quality video and all contributions are freely accessible online.

This section has its own submission website at https://mc.manuscriptcentral.com/e-videos 